\title{
PROMOÇÃO DE ESTRATÉGIAS DE APRENDIZAGEM EM ESTUDANTES DE PSICOLOGIA
}

\author{
Alana Augusta Concesso de Andrade ${ }^{1} \mathbb{D}$; Marisa Cosenza Rodrigues ${ }^{1} \mathbb{D}$; Pedro Paulo Marci Tette ${ }^{1} \mathbb{D}$; Beatriz \\ Maia Soares Silva ${ }^{1} \mathbb{D}$; Bruna Costa de Almeida ${ }^{1} \mathbb{D}$; Humberto dos Reis Pereira ${ }^{1} \mathbb{D}$; Thaynara Barbosa ${ }^{1} \mathbb{D}$; Victória \\ Marques Cândido ${ }^{1} \mathbb{D}$
}

\section{RESUMO}

As estratégias de aprendizagem são processos autorregulatórios de aquisição e uso do conhecimento que devem ser constantemente estimuladas para um amplo aproveitamento da vida acadêmica. O objetivo do estudo foi avaliar a eficácia de um programa de promoção de estratégias de aprendizagem em universitários. Utilizou-se metodologia quasi-experimental com 20 alunos de Psicologia, distribuídos em grupo experimental e controle, sendo realizados préteste, pós-teste e um segundo pós-teste quatro meses após a intervenção. O programa consistiu de oito encontros, focalizando-se a metacognição, administração de recursos internos e contextuais e autorregulação social. O primeiro pós-teste indicou aumento significativo da média do grupo experimental, que se manteve no segundo pós-teste, sendo o principal aumento observado na autorregulação de recursos. O grupo controle não modificou significativamente seu escore total ao longo dos pós-testes e ainda apresentou diminuição significativa na autorregulação social. Os resultados indicam eficácia da intervenção e reforçam a relevância de programas dessa natureza.

Palavras-chave: aprendizagem; autorregulação; ensino superior.

\section{Promotion of learning strategies for psychology students}

\section{ABSTRACT}

Learning strategies are self-regulating processes of knowledge acquisition and use that must be constantly stimulated for the full enjoyment of academic life. The aim of the study was to evaluate the effectiveness of a learning strategy program, which encourage college students. A quasi-experimental methodology was used with 20 Psychology students, distributed in experimental and control groups, being performed pre-test, post-test and a second post-test four months after the intervention. The program consisted of eight meetings, focusing on metacognition, internal and contextual resource management, and social self-regulation. The first post-test indicated a significant increase in the experimental group average, which remained in the second post-test, being the main increase observed in self-regulation of resources. The control group did not significantly change its total score over the post-tests and still showed a significant decrease in social self-regulation. The results indicate the effectiveness of the intervention and reinforce the relevance of such programs.

Keywords: learning; self-regulation; higher education

\section{Promoción de estrategias de aprendizaje en estudiantes de psicología}

\section{RESUMEN}

Las estrategias de aprendizaje son procesos autorregulatorios de adquisición y uso del conocimiento que deben ser constantemente estimuladas para un amplio aprovechamiento de la vida académica. El objetivo del estudio fue evaluar la eficacia de un programa de promoción de estrategias de aprendizaje en universitarios. Se utilizó metodología cuasi-experimental con 20 alumnos de Psicología, distribuidos en grupo experimental y control, siendo realizados pre-test, post-test y un segundo post-test cuatro meses tras la intervención. El programa consistió de ocho encuentros, focalizándose la metacognición, administración de recursos internos y contextuales y autorregulación social. El primer post-test indicó aumento significativo del promedio del grupo experimental, que se mantuvo en el segundo post-test, siendo el principal aumento observado en la autorregulación de recursos. El grupo control no modificó significativamente su escore total a lo largo de los post-testes y aún presentó disminución significativa en la autorregulación social. Los resultados indican eficacia de la intervención y refuerzan la relevancia de programas de esa naturaleza.

Palabras clave: aprendizaje; autorregulación; enseñanza universitaria.

\footnotetext{
1 Universidade Federal de Juiz de Fora - Minas Gerais - MG - Brasil; alana.andrade@gmail.com; rodriguesma@terra.com.br; pedrotette777@gmail.com; smaiaib@gmail.com; bnacalmeida@gmail.com; humbertodrp@gmail.com; thaynarakta@gmail.com; vcandido.psi@gmail.com
} 


\section{INTRODUÇÃO}

No Brasil, a entrada no ensino superior de educação vem crescendo, ultrapassando oito milhões de matrículas em 2.364 Instituições de Ensino Superior (IES), segundo os dados do Instituto Nacional de Estudos e Pesquisas Educacionais Anísio Teixeira (INEP) para o ano de 2015. Contudo, observa-se neste nível de escolaridade um índice considerável de evasões e de mudanças de curso (Instituto Nacional de Estudos e Pesquisas Educacionais Anísio Teixeira [INEP], 2018).

Um processo importante para que o estudante consiga lidar com as dificuldades do contexto universitário é a autorregulação da aprendizagem, também conhecida como aprendizagem autorregulada. De acordo com Zimmerman e Schunk (2011), tal capacidade refere-se à habilidade do aluno de se autogerenciar, por meio de uma atividade proativa em que monitora sua cognição, motivação, comportamento e ambiente, ampliando o desempenho e os resultados da aprendizagem. Dessa forma, adquire habilidades acadêmicas, como definir metas, selecionar e aplicar estratégias, enquanto monitora a própria eficácia.

Uma vez que a aprendizagem autorregulada exige dos estudantes que assumam responsabilidade pelo próprio processo de aprender, ela se torna um tipo desejado de aprendizagem. Além do mais, evidências indicam que a autorregulação é preditora de boa aprendizagem e rendimento acadêmico, sugerindo ser o uso de estratégias autorreguladoras essencial para uma adequada realização de tarefas (Broadbent \& Poon, 2015; Kizilcec, Pérez-Sanagustín, \& Maldonado, 2017; Pascual \& Rosillo, 2015).

Estratégias de aprendizagem atuam como um conjunto de mecanismos que possibilitam que haja a autorregulação da aprendizagem. Elas podem ser definidas como procedimentos de caráter consciente e intencional escolhidos com a finalidade de adquirir, armazenar e utilizar conhecimento ou informação de maneira efetiva em diferentes contextos (Gargallo, Suárez-Rodríguez, \& Pérez-Pérez, 2009; Santos \& Boruchovitch, 2009).

Boruchovitch e Santos (2015) explicam que, apesar de não haver um consenso a respeito da classificação das estratégias de aprendizagem, elas podem ser divididas em três grupos: estratégias cognitivas, estratégias metacognitivas e estratégias de administração de recursos. As estratégias cognitivas envolvem comportamentos que tornam o armazenamento das informações mais eficiente, como habilidades de memorização, elaboração e organização do conteúdo. Já as estratégias metacognitivas são aquelas através das quais o aluno planeja, monitora e regula o próprio pensamento, coordenando a aprendizagem. As estratégias de administração de recursos, por sua vez, certificam a organização do ambiente, a remoção de sentimentos negativos, o aumento da motivação, a redução da ansiedade, entre outras táticas.

Quanto à avaliação de estratégias de aprendizagem, existem instrumentos de referência tanto internacionais (Pintrich \& de Groot, 1990; Zimmermann \& Martinez-Pons, 1986) como nacionais (Boruchovitch et al., 2006; Minervino et al., 2005). Para a sua mensuração no cenário do ensino superior, destaca-se a Escala de Estratégias de Aprendizagem para Estudantes Universitários (EEA-U), criada por Boruchovitch e Santos (2015), a qual apresenta bons parâmetros psicométricos, tornando-a adequada para uso em estudos que avaliam perfil de estratégias e investigam eficácia de intervenção.

Nückles, Rübner e Renkl (2009) evidenciam que há pouco conhecimento por parte de estudantes sobre a utilização apropriada das estratégias de aprendizagem e também sobre como autorregular a própria aprendizagem. Nesse sentido, um estudo realizado por Marini e Boruchovitch (2014) revelou que ocorre um declínio no uso das estratégias conforme o tempo de permanência dos alunos na universidade aumenta. Esse acontecimento vai na contramão do que seria o ideal, uma vez que existem evidências de correlação entre tal utilização e um melhor desempenho acadêmico (Gargallo et al., 2009; Gargallo, Campos, \& Almerich, 2016; Soares, Guisande, Almeida, \& Páramo, 2009).

Mega, Ronconi e De Beni (2014), em uma pesquisa sobre a relação entre emoções, motivação e autorregulação da aprendizagem, encontraram evidências consonantes com os achados iniciais de Pintrich e de Groot (1990). Tal estudo, que é referência sobre o tema, aponta que os alunos conhecerem estratégias de aprendizagem não é suficiente; eles devem ser motivados a utilizá-las e a regular sua cognição e seu esforço. Somente assim, os estudantes se tornariam mais competentes no estudo e melhorariam seu desempenho escolar. Nessa perspectiva, nota-se a relevância da implementação de programas que ensinem tais estratégias para estudantes universitários, uma vez que, como destacado por Costa e Boruchovitch (2010), apesar de existirem produções sobre o tema, o interesse tem se voltado para a investigação a respeito das estratégias em alunos do ensino fundamental e médio.

No que tange os efeitos sobre a utilização de estratégias de aprendizagem em universitários, Montero e Arizmendiarrieta (2017), conduziram um estudo de intervenção com um grupo controle de 57 alunos (sem treinamento) e um grupo experimental com a participação de 60 alunos que cursavam a Faculdade de Formação de Professores e Educação da Universidade de Oviedo na Espanha, os quais foram pré e pós-testados quanto à utilização de estratégias de aprendizagem por meio do Questionário de Estratégias de Aprendizagem e Motivação (Cuestionario de Estrategias de Aprendizaje 
y Motivación - 2ª Revisão - CEAM - R2). A intervenção consistiu em 13 sessões práticas em sala de aula, com duração de duas horas cada, mais trabalhos individuais estimados entre seis e 13 horas. Os resultados evidenciaram um efeito positivo do programa com diferença estatisticamente significativa quanto à utilização de estratégias de aprendizagem dos participantes, quando comparadas as etapas pré e pós-intervenção e os grupos controle e experimental. Ainda que tal mudança não tenha ocorrido em relação a todas as estratégias testadas, os dados sugerem que os estudantes tendem a se beneficiar de programas desse caráter.

Em outro programa, realizado por Rosário et al. (2007), 66 estudantes do primeiro ano da Universidade de Oviedo foram treinados em estratégias de aprendizagem durante seis sessões de uma hora, pré e pós-testados por meio do questionário sobre Conhecimentos das Estratégias de Autorregulação (CEA). Houve melhoria estatisticamente significativa no conhecimento declarativo das estratégias de aprendizagem.

Destaca-se o trabalho de Gargallo e variados colaboradores que, desde o início dos anos 2000 até os dias atuais (Gargallo, 2001; Gargallo et al., 2017), vêm pesquisando a eficácia de programas de intervenção com foco nas estratégias de aprendizagem com universitários. Seu modelo de intervenção inclui aprendizagem vicária, ensino de estratégias de memória e atenção, além de abordar aspectos emocionais da aprendizagem, como motivação e autoconceito. A eficácia do modelo vem sendo evidenciada ao longo do tempo e chegou, em alguns casos, a compor grade curricular de escolas espanholas (Gargallo \& Remensal, 2000).

Gargallo et al. (2016) elaboraram uma disciplina opcional intitulada "Estratégias de aprendizagem e técnicas de estudo para estudantes universitários? a fim de promover estratégias de aprendizagem em 47 estudantes da Universidade de Valencia. Foram oferecidas 14 aulas com duração de duas horas cada. Os participantes foram pré e pós-testados a respeito da utilização de estratégias de aprendizagem através do Questionário de Avaliação das Estratégias de Aprendizagem de Estudantes Universitários (Cuestionario de Evaluación de las Estrategias de Aprendizaje de los Estudiantes Universitarios - CEVEAPEU). Os resultados evidenciaram diferenças significativas nas estratégias de aprendizagem, assim como no desempenho acadêmico daqueles que cursaram a disciplina oferecida, o que não aconteceu com o grupo controle.

No Brasil, algumas pesquisas têm buscado investigar as estratégias mais utilizadas pelos universitários, mostrando que autorregulação de recursos e estratégias cognitivas são preferidas por eles (Góes, Pavesi, \& Alliprandini, 2013; Mello, 2017; Silva, 2012). No que diz respeito às intervenções, ressalta-se a investigação de Polydoro, Pelissoni, Carmo, Emílio, Dantas e Rosário (2015) que detectaram eficácia de um programa de intervenção, em formato de disciplina, para 124 universitários que responderam a um questionário avaliativo no final do curso. A disciplina abordou gerenciamento de tempo, planejamento, memória, controle de ansiedade, entre outros temas e, como resultado, os estudantes relataram mudanças significativas na rotina de estudo e nas estratégias adotadas para aprender.

Os ganhos principais de intervenções em estratégias de aprendizagem são promoção, prevenção e remediação das dificuldades escolares. Como apresentado, a literatura científica aponta diversas experiências de sucesso na intervenção em estratégias de aprendizagem, independente da abordagem teórica adotada. Rosário et al. (2015) afirmam que tais programas de intervenção são eficazes com alunos de diferentes origens culturais, linguísticas e educacionais.

Os indicadores positivos dos estudos mencionados permitem considerar o quanto é desejável que a universidade proporcione aos alunos programas que visem promover o ensino de estratégias de aprendizagem. Diante do exposto, essa investigação propõe avaliar o efeito de um programa de promoção de estratégias de aprendizagem em uma amostra de conveniência composta por alunos de Psicologia de uma universidade pública no estado de Minas Gerais. A intervenção em tal contexto se torna útil, uma vez que acolhe uma população de universitários crescente no país e que também apresenta necessidades escolares a serem atendidas.

\section{MÉTODO}

\section{Delineamento}

A fim de testar o efeito de intervenção, utilizou-se metodologia quasi-experimental, mediante uso de grupo experimental (GE) e de grupo controle (GC) não equivalente. Ambos os grupos participaram de um pré-teste, um pós-teste e de um segundo pós-teste quatro meses após o fim da intervenção. Os dados coletados foram predominantemente quantitativos com registro qualitativo do decorrer das sessões. A amostragem se deu de forma intencional, não probabilística e por conveniência, ou seja, todos os participantes eram graduandos de Psicologia, não havendo aleatorização dos componentes nos grupos e, principalmente, em que se contou com o interesse dos alunos em participar da intervenção. Essa forma de seleção de participantes foi necessária para que fosse garantida a adesão do grupo ao programa de promoção desde seu início até o fim.

\section{Participantes}

O GE $(N=10)$ foi composto por sete mulheres e três homens com média de idade de 20,50 anos ( $D P=0,972$ ). Já o GC $(N=10)$ foi formado por oito mulheres e dois homens com média de idade de 19,80 anos $(D P=1,22)$. 
Todos os participantes eram alunos do curso de Psicologia de uma universidade pública de uma cidade da Zona da Mata Mineira.

\section{Instrumentos}

Escala de Estratégias de Aprendizagem para Universitários (EEA-U)

A EEA-U foi criada por Boruchovitch e Santos (2015), constituindo-se de um autorrelato com 35 itens que avaliam objetivamente a maneira como estudantes universitários aprendem e estudam. As afirmações são respondidas por meio de escala Likert de quatro pontos que variam entre nunca $=1$ e sempre $=4$. As autoras utilizaram a análise fatorial exploratória aplicada em dados de ampla amostra universitária $(N=1.490)$ e identificaram três estratégias de aprendizagem: 1 ) estratégias autorregulatórias cognitivas e metacognitivas; 2) estratégias de autorregulação de recursos internos e contextuais e; 3 ) estratégias de autorregulação social. A estatística de precisão por Alpha de Cronbach indicou um índice bastante satisfatório para o total da escala $(\alpha=0,87)$.

\section{Diário de Campo}

A cada encontro foram registradas observações sobre o andamento do grupo mediante dois eixos de observações: 1) as manifestações do grupo demonstradas por meio de reflexões e comentários sobre os conteúdos treinados e; 2 ) relatos sobre a utilização das estratégias no cotidiano acadêmico durante a implementação do trabalho.

\section{Procedimentos}

Após aprovação do projeto pelo Comitê de Ética em Pesquisa da universidade onde ocorreu o estudo (CAAE 67671817.5.0000.5147), iniciou-se a etapa de coleta de dados. Realizou-se uma ampla divulgação do projeto para todos os alunos do curso de Psicologia, obtendo-se a adesão de alguns alunos voluntários que assinaram o Termo de Consentimento Livre Esclarecido (TCLE) e respondendo em seguida a EEA-U, procedimentos que envolveram em média 20 minutos. Os participantes foram alocados nos grupos GE e GC, por critério de interesse e conveniência, uma vez que não houve randomização da amostra. Contudo, a fim de manter uma conduta ética de oferta de benefícios para todos os grupos da pesquisa, foi realizada uma palestra sobre estratégias de aprendizagem ao GC, com duração de 60 minutos.

A intervenção com o GE se deu em oito encontros, uma vez por semana, com duração de cerca de 90 minutos cada um. As sessões aconteceram em uma sala da própria instituição de ensino, fora do horário regular de aulas dos alunos participantes. O programa de promoção foi coordenado por alunos bolsistas integrantes do Programa de Educação Tutorial (PET) do curso de Psicologia da universidade onde aconteceu a pesquisa e por uma bolsista de iniciação científica, os quais foram capacitados e supervisionados pela docente tutora do PET e por uma docente colaboradora. A cada encontro um desses alunos realizava o registro do diário de campo. Ao término da intervenção, realizou-se o primeiro pós-teste com a EEA-U no GE e no GC. Quatro meses depois, aplicou-se o segundo pós-teste em ambos os grupos visando identificar, em uma avaliação de seguimento, a possível manutenção de habilidades treinadas. As avaliações ocorreram em sala, de forma coletiva e nas dependências da instituição.

Procedimentos da intervençãoOs encontros para estimulação das estratégias de aprendizagem foram baseados em programas testados e publicados pela literatura científica internacional, mais especificamente, no programa publicado na íntegra por Gargallo e Remensal (2000) e atualizado em publicações posteriores (Gargallo et al., 2016; Gargallo, Esteban, Mateo, Peleato, \& Rodríguez, 2015). Buscou-se manter a metodologia de intervenção do trabalho citado, realizando poucas alterações, como número de encontros, adaptação dos temas para o curso de Psicologia e busca de equivalência entre os conteúdos estimulados nos encontros e os construtos avaliados pela escala adotada na pesquisa. Cada encontro foi iniciado com uma aula expositiva sobre o tema do dia, seguida de uma vivência prática e finalizado com estabelecimento de tarefa de casa, que passou a ser checada a partir do segundo encontro.

O primeiro e o segundo encontros objetivaram o exercício da metacognição, incentivando a reflexão dos alunos sobre a própria aprendizagem. Houve uma palestra e um debate sobre estratégias de aprendizagem e os participantes foram convidados a autoavaliar sua motivação e autoconceito, relacionando esses construtos com seus objetivos acadêmicos. $\mathrm{O}$ terceiro encontro abordou condições de estudo, atenção e concentração por meio de leitura e imaginação de estórias e também uma vivência para estimular a atenção a detalhes. No quarto encontro, os coordenadores do grupo apresentaram formas de montar agenda de estudo e aplicaram técnicas de relaxamento, focalizando-se, nessas duas sessões, a autorregulação de recursos internos e contextuais. O quinto e o sexto encontros visaram promover habilidades de apreensão de leitura com uso de táticas como resumir, sublinhar e desenhar mapas conceituais, sendo utilizados textos de Psicologia. O sétimo encontro dedicou-se a treinar recursos de memória, a partir de uma sensibilização sobre construção de memória significativa. Foram propostos exercícios com lista de palavras, de imagens e recuperação de detalhes de estórias contadas. Portanto, com essas sessões, buscou-se exercitar capacidades cognitivas e metacognitivas da aprendizagem. $\mathrm{O}$ oitavo encontro e último tratou de habilidades sociais, mais especificamente, resolução de conflitos e tomada de decisões, que foram estimuladas por meio de análise de estórias de conflitos, procurando- 
-se chegar a um acordo para resolvê-los. Também foram estimuladas habilidades de comunicação verbal e não verbal, promovendo assim, a desevoltura social exigida para uma série de tarefas acadêmicas que envolvem a interação em grupo.

\section{ANÁLISE DE DADOS}

As análises descritivas e inferenciais foram realizadas por meio do programa Statistic Package of Social Sciences (SPSS) da International Business Machines (IBM) versão 21. Optou-se pelo uso de estatísticas não paramétricas, visto que a amostra é pequena e com pouca variabilidade. $O$ teste Wilcoxon foi aplicado para detectar diferenças intragrupo e o teste Mann-Whitney para as diferenças intergrupo. Alguns cuidados foram tomados para além do nível de significância, como o cálculo do tamanho do efeito ( $d$ de Cohen), que determina a área de distância entre as médias dos grupos, e a estimativa de poder estatístico, que leva em conta tamanho amostral, tamanho do efeito e nível de significância assumido (Hair, Black, Babin, Anderson, \& Tatham, 2009). $O$ tamanho do efeito e o poder estatístico foram computados no software GPower versão 3.1.9.2.

\section{RESULTADOS E DISCUSSÃO}

\section{Análise intragrupo}

A análise de dados se baseou no desempenho dos participantes em cada uma das três subescalas e no escore total da EEA-U em três momentos de testagens: pré-teste, primeiro pós-teste e segundo pós-teste. A maneira como os itens de cada subescala foram somados seguiu o que é sugerido no trabalho de validade fatorial publicado pelas autoras do instrumento (Boruchovitch \& Santos, 2015). A Tabela 1 apresenta média e desvio-padrão desses resultados para GE $(N=10)$ e GC $(N=10)$.

$O$ teste estatístico Wilcoxon aplicado ao escore total obtido pelo GE na EEA-U, em análise pareada com o pré-teste, encontrou aumento significativo no primeiro pós-teste $(z=-1,836 ; p=0,036) ; d=0,43$ e poder de $35,2 \%$; no segundo pós-teste com nível de $p$ próximo da significância estatística $(z=-1,602 ; p=0,057) ; d=0,30$ e poder de $31,8 \%$. Apesar de leve queda de quase dois pontos na média do GE no estudo de seguimento, a comparação entre primeiro e segundo pós-teste não encontrou diferença significativa $(z=-0,153 ; p=0,453)$. Assim sendo, mesmo com pequeno efeito e poder encontrados, 0 resultado significativo permite afirmar que houve aumento de desempenho do GE após intervenção, o qual se manteve no final do estudo.

Em relação ao desempenho do GE nas subescalas da EEA-U, a autorregulação de recursos internos $e$ contextuais apresentou aumento significativo quando comparada aos resultados de pré-teste com o primeiro pós-teste $(z=-1,781 ; p=0,049)$ com $d=0,65$ e poder de $82,2 \%$; e com o segundo pós-teste $(z=-1,794 ; p=0,043)$ com $d=0,62$ e poder de $75,5 \%$. O tamanho do efeito demonstrado pelo $d$ de Cohen é considerado médio e o poder indica uma alta probabilidade de aceitação de hipótese da pesquisa, ou seja, eficácia da intervenção nessas habilidades específicas dos sujeitos estudados e nesse período de tempo.

A comparação entre primeiro e segundo pós-teste não revelou mudança significativa $(z=-0,316$; $p=0,434$ ), indicando manutenção do aumento de tais habilidades durante os meses da coleta. Os resultados permitem indicar que a intervenção apresentou efeitos relativamente duradouros corroborando, por exemplo, com os estudos de Fernandes e Frison (2015) e Lai e Hwang (2016).

Considera-se possível que o incremento obtido no GE quanto ao escore total da escala se deva à melhoria na autorregulação de recursos internos e contextuais, uma vez que as demais subescalas, apesar do aumento de médias, não apresentaram mudanças significativas ao nível de $p \leq 0,05$. Portanto, a intervenção parece ter

Tabela 1. Média e desvio-padrão de GE e GC nas subescalas e no escore total da EEA-U

\begin{tabular}{|c|c|c|c|c|c|c|}
\hline \multirow{2}{*}{ Grupo experimental $(N=10)$} & \multicolumn{2}{|c|}{ Pré-teste } & \multicolumn{2}{|c|}{ Primeiro pós-teste } & \multicolumn{2}{|c|}{ Segundo pós-teste } \\
\hline & $M$ & $D P$ & $M$ & $D P$ & $M$ & $D P$ \\
\hline Cognitivas/metacognitivas & 66,60 & 8,79 & 67,60 & 8,38 & 68,70 & 8,13 \\
\hline Internas/contextuais & 22,30 & 3,68 & 24,33 & 2,50 & 24,30 & 2,71 \\
\hline Sociais & 10,80 & 2,97 & 10,80 & 2,89 & 10,40 & 2,50 \\
\hline Escore total & 99,70 & 12,99 & 105,30 & 12,91 & 103,50 & 12,43 \\
\hline Grupo controle $(N=10)$ & $M$ & $D P$ & $M$ & $D P$ & $M$ & $D P$ \\
\hline Cognitivas/metacognitivas & 63,50 & 10,49 & 63,22 & 10,44 & 62,70 & 11,45 \\
\hline Internas/contextuais & 23,30 & 4,13 & 24,10 & 3,87 & 22,90 & 5,23 \\
\hline Sociais & 11,10 & 1,91 & 10,00 & 1,82 & 9,50 & 1,95 \\
\hline Escore total & 97,90 & 12,46 & 95,60 & 12,25 & 94,20 & 16,27 \\
\hline
\end{tabular}

Nota. EEA-U: Escala de estratégias de aprendizagem para universitários; GE: grupo experimental; GC: grupo controle. 
abordado adequadamente as estratégias mencionadas, auxiliando esses participantes a lidarem com a ansiedade diante das tarefas acadêmicas e a organizar melhor seu ambiente de trabalho durante a realização da pesquisa. As estratégias de autorregulação metacognitiva e cognitiva e de autorregulação social não aumentaram de forma crítica. A intervenção tratou amplamente dos temas relacionados à cognição e à metacognição, mas talvez fosse necessário ampliar o número de encontros, visando alterar mais profundamente as habilidades do espectro. A dificuldade de se aumentar significativamente as capacidades cognitivas é relatada pela literatura (Melby-Lervag, Redick, \& Hulme, 2016). Já a autorregulação social foi tema apenas da última sessão que focalizou aspectos das habilidades sociais levemente diferentes daqueles avaliados pela EEA-U. A escala adotada possui quatro itens que avaliam o fator social, os quais se referem às estratégias de busca de ajuda no grupo. Assim sendo, o não aumento dessas características pelo GE pode se dever a uma pequena incongruência entre o que foi treinado e o que foi mensurado pelo instrumento.

No que tange ao GC $(N=10)$, a análise de Wilcoxon aplicada ao escore total da escala não encontrou diferenças significativas entre pré-teste, primeiro pós-teste $(z=-1,424 ; p=0,091)$ e segundo pós-teste $(z=-1,020$; $p=0,163)$. Também não foram encontradas diferenças significativas entre primeiro e segundo pós-teste ( $z=-$ $0,869 ; p=0,214)$, ou seja, o grupo que não recebeu intervenção não alterou significativamente seu escore da EEA-U ao longo das testagens.

Quando se estudam as mudanças do GC $(N=10)$ nas subescalas da EEA-U, nota-se uma redução significativa no escore de autorregulação de recursos sociais. Assim sendo, partindo do pré-teste, o escore diminuiu significativamente no primeiro pós-teste $(z=-1,980 ; p=0,035)$, com $d=0,59$ (efeito médio) e poder de $56,4 \%$; no segundo pós-teste $(z=-1,761 ; p=0,056)$ com nível de $p$ quase siginificativo; $d=0,27$ (efeito pequeno) e poder alto de $87,6 \%$. Não houve diferença significativa entre primeiro e segundo pós-teste $(z=-0,689 ; p=0,282)$, apesar da queda observada na média (ver Tabela 1). As demais subescalas não apresentaram alterações significativas ao nível de $p \leq 0,05$.

O GC não evoluiu como o GE e ainda perdeu pontuação na EEA-U durante os pós-testes. Há discussão na literatura sobre o declínio de uso de estratégias de aprendizagem entre universitários ao longo do curso, a qual parece acontecer por falta de motivação ou pelo fato dos alunos terem se acostumado com a rotina de estudos da universidade e, portanto, já não se esforçam tanto para atingir resultados acadêmicos (Marini \& Boruchovitch, 2014). Contudo, os resultados aqui encontrados, em função do tamanho da amostra de 20 participantes, devem ser vistos com cautela.

\section{Análise intergrupo}

A análise de diferenças intergrupais, com uso da estatística Mann Whitney, evidencia que não houve diferenças significativas no pré-teste entre $G C(N=10)$ e GE $(N=10)$ com U de Mann Whitney de 40,50 ( $z=-$ $0,719 ; p=0,247)$, mostrando que os grupos estavam equivalentes no nível de estratégias de aprendizagem antes da intervenção. No primeiro pós-teste após a intervenção, o GE apresentou escore médio mais alto que o GC, conforme se observa na Tabela 1. No entanto, encontrou-se nível de $p$ apenas marginal $(z=-1,563 ; p=0$, 065) com U de Mann Whitney = 29,50; tamanho de efeito grande $(d=0,77)$ e poder bastante alto de $85,6 \%$. $\mathrm{O}$ grande efeito encontrado pelo $d$ de Cohen e o alto poder estatístico indicam que as médias dos grupos realmente se diferem e que a hipótese da pesquisa deve ser aceita. Assim sendo, o GE superou o GC em estratégias de aprendizagem no primeiro pós-teste. $A$ mesma análise aplicada ao segundo pós-teste não alcançou o nível de $p \leq 0,05(z=-1,286 ; p=0,106)$, apesar de $\mathrm{GE}(M=103,50 ; D P=12,43)$ ter apresentado escore médio mais alto que o $\mathrm{GC}(M=94,20 ; D P=16,27)$ em quase dez pontos na avaliação final. $O$ tamanho do efeito para esta análise foi médio $(d=0,64)$ e poder do teste aceitável com $78 \%$. Portanto, é possível afirmar que as médias dos grupos ao final do estudo são verdadeiramente diferentes e favorecem o GE, ou seja, a vantagem de GE sobre GC perdurou ao longo do intervalo de tempo no seguimento.

Conclui-se que a diferença encontrada entre os grupos se deu provavelmente em função da intervenção. Resultados de eficácia de intervenção em estratégias de aprendizagem, com uso de grupo controle, têm sido reportados por pesquisadores da área (Hattie, 2015), porém com um número maior de participantes. Assim como há relatos de que não ocorreram diferenças significativas para o grupo controle, as quais têm sido geralmente atribuídas ao número escasso e descontínuo de sessões realizadas (Gargallo \& Remensal, 2000).

Com relação às subescalas, a estatística descritiva mostrou que o GE obteve escores mais altos que GC nas subescalas nos pós-testes da pesquisa. Todavia, a análise Mann Whitney aplicada aos escores obtidos pelos grupos nos três momentos de testagem não encontrou diferenças significativas entre GE $(N=10)$ e GC $(N=10)$, sendo os valores de $p$ encontrados todos superiores a 0,05 . Dessa maneira, em comparação com o grupo controle, os ganhos do GE foram no escore total da EEA-U e não nos escores específicos.

Em suma, as análises estatísticas intra e intergrupo mostraram efeito significativo da intervenção ao longo dos meses de coleta, sendo esse efeito demonstrado tanto no escore total, quanto em algumas subescalas da 
EEA-U. O resultado também foi mais significativo, com maior poder e tamanho de efeito no nível intragrupal em escores específicos, mostrando avanço de GE e retrocesso de GC. Ao passo que as diferenças nos escores totais foram mais fortes no nível intergrupal, também em favor do GE.

\section{Diário de campo}

Os estudos psicológicos de intervenção com pequenas amostras nem sempre alcançam a significância estatística esperada, mas podem gerar significância psicológica (Dancey \& Reidy, 2008). Por esta razão, é importante utilizar registros qualitativos de dados, como o diário de campo, para complementar os dados quantitativos. O registro do diário de campo deste estudo de intervenção indicou que a maior parte do grupo se mostrou curioso, motivado e participativo. Os participantes tiravam dúvidas, realizavam os exercícios propostos em sala e faziam anotações durante as intervenções. Observou-se também, nos feedbacks orais do grupo, que o programa estava sendo positivo, visto que alguns universitários relataram ter começado a utilizar as estratégias no cotidiano das aulas da graduação.

\section{CONSIDERAÇÕES FINAIS}

A presente pesquisa almejou promover estratégias de aprendizagem em estudantes de Psicologia. Os resultados encontrados mostraram que os alunos investigados se beneficiaram da intervenção durante os meses de realização do estudo, aumentando suas habilidades de aprendizagem, conforme mensurada pela escala e relatando verbalmente o benefício do programa no dia a dia da vida acadêmica. Por outro lado, o grupo que não usufruiu da intervenção, apresentou diminuição significativa na autorregulação, sinalizando que, caso as habilidades não sejam continuamente estimuladas, elas podem declinar ao longo do tempo. Dessa maneira, pesquisas e intervenções na área devem ser incentivadas.

$O$ estudo apresenta limitações. A principal delas diz respeito ao tamanho limitado da amostra que foi voluntária e dependeu da adesão do grupo universitário o que indica que os resultados devem ser vistos com a devida cautela. Recomenda-se portanto, para futuros estudos dessa natureza a ampliação da amostra bem como a realização de mais encontros de intervenção ou torná-los contínuos. Além disso, ressalta-se que o trabalho foi realizado por alunos de graduação que, embora devidamente supervisionados, podem não ter apresentado a experiência necessária para estimular um grupo composto por seus próprios pares. Daí a importância de envolver docentes e profissionais na realização do trabalho. Nesse sentido, sugere-se inserir intervenções em estratégias de aprendizagem nos currículos das universidades, bem como capacitar professores para estimular seus alunos na sala de aula durante as disciplinas. Aponta-se também a necessidade de autoavaliação contínua e contextualizada das habilidades para analisar melhor o impacto de intervenções.

Considera-se que ampliando o número de participantes, tornando a amostra mais representativa da população universitária e alongando o programa no tempo poderá ser possível traçar conclusões mais gerais sobre quais estratégias de aprendizagem são mais adequadas para a maioria dos estudantes e quais delas podem ser promovidas pelos professores em sala de aula, sempre levando em conta que estratégias de aprendizagem não são habilidades que possam ser padronizadas e/ou homogeneizadas, tornando-se necessário respeitar as preferências do aluno ao estudar e aprender.

ão há dúvidas de que a estimulação das estratégias de aprendizagem leva os alunos a aproveitarem melhor os conhecimentos adquiridos na graduação, maximizando o rendimento acadêmico e contribuindo para uma melhor inserção no mercado de trabalho. No entanto, ainda é necessário superar o caráter excessivamente clínico e remediativo dos programas de intervenção e fazer com que a promoção de estratégias seja intrínseca ao próprio plano curricular das instituições de ensino. Espera-se que os achados aqui reportados também possam fomentar discussão para outras fases da vida escolar.

\section{REFERÊNCIAS}

Boruchovitch, E.; Santos, A. A. A. (2015). Psychometric studies of the learning strategies scale for university students. Paidéia, 25(60), 19-27. http://dx.doi.org/10.1590/198243272560201504

Boruchovitch, E.; Santos, A. A. A.; Costa, E. R.; Neves, E. R. C.; Cruvinel, M.; Primi, R.; Guimarães, S. E. R. (2006). A construção de uma escala de estratégias de aprendizagem para alunos do ensino fundamental. Psicologia: Teoria e Pesquisa, 22(3), 297-304. http://dx.doi.org/10.1590/ S0102-37722006000300006.

Broadbent, J.; Poon, W. L. (2015). Self-regulated learning strategies and academic achievement in online higher education learning environments: a systematic review. Internet \& Higher Education, 27, 1-13. http:// dx.doi.org/10.1016/j.iheduc.2015.04.007.

Costa, E. R.; Boruchovitch, E. (2010). As estratégias de aprendizagem de alunos repetentes do ensino fundamental. Psicologia em Pesquisa, 4(1), 31-39. http://pepsic. bvsalud.org/scielo.php?script=sci_arttext\&pid=S1982$12472010000100005 \&$ lng=pt\&tlng=pt.

Dancey, C. P.; Reidy, J. (2008). Estatística sem matemática para Psicologia. Usando SPSS para Windows. Porto Alegre: Artmed. Bookman.

Fernandes, V. R.; Frison, L. M. B. (2015). Estratégias de aprendizagem autorregulatória no ensino superior: escrita de um artigo científico. Psicologia da Educação, 41, 37-49. http://dx.doi.org/10.5935/2175-3520.20150013.

Gargallo, B. (2001). Learning strategies. A training programme. 
European Journal of Psychology of Education, 16, 49-65. https://www.jstor.org/stable/23420414

Gargallo, B.; Campos, C.; Almerich, G. (2016). Learning to learn at university. The effects of an instrumental subject on learning strategies and academic achievement. Cultura y Educacion, 28(4), 771-810. https://doi.org/10.1080/113 56405.2016.1230293.

Gargallo, B.; Esteban, P. R. G.; Mateo, P. M. S.; Peleato, I. V.; Rodríguez, M. A. J. (2015). Métodos centrados en el aprendizaje, estrategias y enfoques de aprendizaje en estudiantes universitarios. Revista de Educación, 370, 2292540. doi: 10.4438/1988-592X-RE-2015-370-304.

Gargallo, B.; Pérez, C. P.; Rodríguez, M. A. J.; Hervás, N. M.; Beut, J. A. G. (2017). Métodos centrados en el aprendizaje, implicación del alumno y percepción del contexto de aprendizaje em estudiantes universitarios. Educación XX1, 20, 161-187. http://revistas.uned.es/index.php/ educacionXX1/article/view/19036

Gargallo, B.; Remensal, A. (2000). Estratégias de aprendizaje. Un programa de intervención para ESO y EPA. Madrid: Ministério da Educación, Cultura y Deporte.

Gargallo, B.; Suárez-Rodríguez, J.; Pérez-Pérez, C. (2009). The CEVEPEAU questionnaire. An instrument to assess the learning strategies of university students. Revista Electrónica de Investigación y Evaluación Educativa, 15(2), 1-26. http:// www.uv.es/RELIEVE/v15n2/RELIEVEv15n2_5eng.htm.

Góes, N. M.; Pavesi, M. A.; Alliprandini, P. M. Z. (2013). Estratégias de aprendizagem utilizadas por alunos do curso de Pedagogia de uma IES pública do estado do Paraná ofertado à distância. RENOTE Revista Novas Tecnologias na Educação, 11. https://doi.org/10.22456/1679-1916.44737

Hair, J. F.; Black, W. C.; Babin, B. J.; Anderson, R.E.; Tatham, R.L. (2009). Análise multivariada de dados (6a ed.). Porto Alegre: Bookman.

Hattie, J. (2015). The applicability of Visible Learning to higher education. Scholarship of Teaching and Learning in Psychology, 1(1), 79-91. http://dx.doi.org/10.1037/ stl0000021.

Instituto Nacional de Estudos e Pesquisas Educacionais Anísio Teixeira [INEP] (2018). Resumo técnico do censo da educação superior 2015. Brasília: INEP, Ministério da Educação. Recuperado de http://portal.inep.gov.br/censoda-educacao-superior.

Kizilcec, R. F.; Pérez-Sanagustín, M.; Maldonado, J. J. (2017). Self-regulated learning strategies predict learner behavior and goal attainment in Massive Open Online Courses. Computers \& Education, 104, 18-33. https://doi. org/10.1016/j.compedu.2016.10.001

Lai, C. L.; Hwang, G. J. (2016). A self-regulated flipped classroom approach to improving students' learning performance in a mathematics course. Computers \& Education, 100, 126140. http://dx.doi.org/10.1016/j.compedu.2016.05.006.

Marini, J.; Boruchovitch, E. (2014). Self-regulated learning in students of pedagogy. Paidéia, 24(59), 323-330. http:// dx.doi.org/10.1590/1982-43272459201406.
Melby-Lervag, M.; Redick, T. S.; Hulme, C. (2016). Working memory training does not improve performance on measures of intelligence or other measures of "far transfer": evidence from a meta-analytic review. Perspectives on Psychological Science, 11(4), 512-534. doi: 10.1177/1745691616635612.

Mega, C.; Ronconi, L.; De Beni, R. (2014). What makes a good student? How emotions, self-regulated learning, and motivation contribute to academic achievement. Journal od Educational Psychology, 106(1), 121-131. http://dx.doi. org/10.1037/a0033546.

Mello, C. G. (2017). Estratégias de aprendizagem em ações educacionais à distância: relação com características da clientela e reações ao curso. Dissertação de mestrado, Programa de pós-graduação de Psicologia da Universidade de São Paulo, São Paulo, SP.

Minervino, C. A. S. M.; Silveira, N. J. D.; Figueiredo, A. A. F.; Oliveira, K. A.; Silva, E. R.; Rodrigues, S. G. (2005). Estudo de validação da escala de estratégias de estudo. Avaliação Psicológica, 4(2), 115-123. http://pepsic. bvsalud.org/scielo.php?script=sci_arttext\&pid=S167704712005000200004\&lng=pt\&tlng=pt.

Montero, C. R.; Arizmendiarrieta, B. S. (2017). The effectiveness of a learning strategies program for university students. Psicothema, 29(4), 527-532. doi: 10.7334/ psicothema2016.171

Nückles, M.; Rübner, S.; Renkl, A. (2009). Enhancing selfregulated learning by writing learning protocols. Learning and Instruction, 19, 259-271. https://doi.org/10.1016/j. learninstruc.2008.05.002.

Pascual, L. R.; Rosillo, V. M. (2015). Efectividad del coaching grupal sobre el desarrollo de la autorregulación del aprendizaje en estudiantes de ingeniería. Cuadernos de Investigación Educativa, 6(1), 71-88. https://revistas.ort. edu.uy/cuadernos-de-investigacion-educativa/article/ view $/ 8 / 6$

Pintrich, P. R.; de Groot, E. V. (1990). Motivational and selfregulated learning components of classroom academic performance. Journal of Educational Psychology, 82(1), 33-40. http://dx.doi.org/10.1037/0022-0663.82.1.33.

Polydoro, S. A. J.; Pelissoni, A. M. S.; Carmo, M. C.; Emílio, E. R. V.; Dantas, M. A.; Rosário, P. (2015). Promoção da autorregulação da aprendizagem na universidade: percepção do impacto de uma disciplina eletiva. Revista de Educação PUC-Campinas, 20, 201-213. https://doi. org/10.24220/2318-0870v20n3a2877.

Rosário, P.; Núñes, J. C.; Trigo, L.; Guimarães, C.; Fernandez, E.; Cerezo, R.; Fuentes, S.; Orellana, M.; Santibáñez, A.; Fulano, C.; Ferreira, A.; Figueiredo, M. (2015). Transcultural analysis of the effectiveness of a program to promote selfregulated learning in Mozambique, Chile, Portugal, and Spain. Higher Education Research \& Development, 34(1), 173-187. https://doi.org/10.1080/07294360.2014.935932

Rosário, P.; Mourão, R.; Núñez, J. C.; González-Pienda, J.; Solano, P.; Valle, A. (2007). Eficacia de un programa instruccional para la mejora de procesos y estrategias de aprendizaje en la enseñanza superior. Psicothema, 19(3), 
422-427. http://www.psicothema.com/pdf/3380.pdf

Santos, O. J. X.; Boruchovitch, E. (2009). Estratégias de aprendizagem na formação dos professores: Uma análise da produção científica. Educação, 32(3), 346-354. http:// revistaseletronicas.pucrs.br/ojs/index.php/faced/article/ view/5349/4206.

Silva, L. L. V. (2012). Estilos e estratégias de aprendizagem de estudantes universitários. Dissertação de mestrado, Programa de pós-graduação em Psicologia da Universidade de São Paulo, São Paulo, SP.

Soares, A. P.; Guisande, M. A.; Almeida, L. S.; Páramo, M.
(2009). Academic achievement in first-year Portuguese college students: The role of preparation and learning strategies. International Journal of Psychology, 44, 204-212. https://doi.org/10.1080/00207590701700545

Zimmerman, B. J.; Martinez-Pons, M. (1986). Development of a structured interview for assessing student use of self-regulated learning strategies. American Educational Research Journal, 23(4), 614-628. http://dx.doi.org/ 10.3102/00028312023004614.

Zimmerman, B. J.; Schunk, D. (2011). Handbook of selfregulation of learning and performance. New York: Taylor \& Francis.

Os autores agradecem o apoio financeiro que a pesquisa recebeu da Pró-reitoria de Pós-graduação e Pesquisa da Universidade Federal de Juiz de Fora (Propp - UFJF) e do Programa de Educação Tutorial do Ministério da Educação (PET - MEC).

Recebido: 23 de agosto de 2018

Aprovado: 29 de outubro de 2019 